\section{Buchrezension zu: Sternstunden der Wissenschaft Eine Erfolgsgeschichte des Denkens}

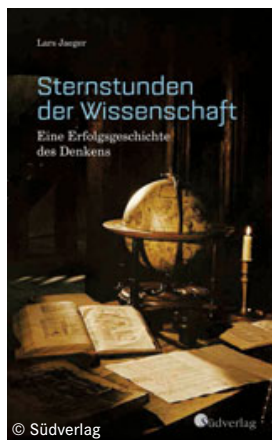

Sternstunden der

Wissenschaft

\section{Eine Erfolgsgeschichte des}

Denkens

Lars Jaeger

335 S., Südverlag, 2020. HC, 20,- $€$. ISBN: 9783878001409

DOI: $10.1007 / \mathrm{s} 12268-020-1498-8$ (C) Springer Verlag GmbH 2020

Die Evolution des Lebens auf der Erde verläuft ungleichmäßig über die Zeit, mitunter in Sprüngen, die häufig auch heute noch unverstanden sind. Auch unser Verständnis der Natur steigt nicht kontinuierlich, erlebt immer wieder plötzlichen Fortschritt, eben ,Sternstunden'. Vielfach sind sie ausgelöst von einzelnen Persönlichkeiten oder Gruppen von Denkern und Wissenschaftlern. Geschichte der Wissenschaft ist Menschheitsgeschichte. Sie darzustellen erfordert Überblick, Detailkenntnis, Fähigkeit zur Zusammenschau. Lars Jaeger gelingt hier eine solche. Er beschreibt den großen Erkenntniszuwachs von der Antike bis heute anhand von Persönlichkeiten, von ,Stars“ am Sternenhimmel der Menschheitsgeschichte, verliert sich dabei aber nicht in Details. Er sieht große zugrunde liegende Tendenzen, wie z.B. den Übergang von den Mythen und Dogmen der Vorund Frühgeschichte zu exaktem Beobachten und Experimentieren, hin zum Forschen.

Lars Jaeger stellt sein Buch unter ein Motto, formuliert vm Phy- siknobelpreisträger Richard Feynmann: Religion ist eine Kultur des Glaubens, Wissenschaft ist eine Kultur des Zweifels. Sternstunden der Wissenschaft sind das Auftreten von Zweiflern an Mythen und Göttern, von Wissenschaftlern in unserem heutigen Sinn. Der Grieche Eratosthenes z.B. legte einen Grundstein für die Abwendung von einem Bild des Kosmos, das die Erde als Scheibe und den Götterhimmel darüber sah. Er beobachtete die ungleiche Länge eines Schattens am Tag der Sommersonnenwende in Alexandria und in Oberägypten. Daraus schloss er auf die Kugelform der Erde und berechnete schon im zweiten vorchristlichen Jahrhundert ziemlich genau den Umfang der Erdkugel. Sein Freund Archimedes aus dem hellenistischen Syrakus steht für Jaeger für die zweite Voraussetzung (neben dem Zweifel an Mythen und Göttern) der ,Erfolgsgeschichte des Denkens': das Auftreten der Mathematik als Werkzeug exakter Erkenntnis. Zweifelndes Beobachten und Mathematik, dies sind die beiden Grundlagen der sich beschleunigenden Entwicklung der (Natur-)Wissenschaften.

Lars Jaegers Werk enthält eine Fülle von, Sternstunden', angereichert um Anekdoten und Lebensläufe. Und um die großen Entwicklungslinien, wie z.B. den Umweg antiken Wissens und Denkens über die arabische Welt in das christliche Abendland. Er schildert Wurzeln der Renaissance wie z.B. die Wiederentdeckung des großen Gedichts De rerum natura des Epikureers Lukrez (99-55 v. Chr.) in einer Klosterbibliothek in Fulda durch den Florentiner Humanisten Poggio Bracciolini (1380-1459). Es enthält so erstaunliche bis in unsere Tage gültige Ideen vom Aufbau der Natur aus winzigen festen Teilchen. Die Spur der Sternstunden belegt er mit den Namen der Großen, von Ptolemäus über Kopernikus, Kepler, Galilei, Newton, Leibniz, bis zu Einstein.
Schwerpunkt sind die Kosmologen und Mathematiker, er vergisst aber auch Galen, Darwin und Harvey nicht. Und der promovierte Physiker, Philosoph und Unternehmer (!) Jaeger zieht die Sternenspur bis zu Anwendern der Naturerkenntnis, die wie der Erfinder der Dampfmaschine James Watt die Grundlagen für den Wohlstand des Abendlandes legten.

Ein spannendes ,buntes“ Buch, gut zu lesen, sehr zu empfehlen!

Ferdinand Hucho, Berlin, hucho@chemie.fu-berlin.de 